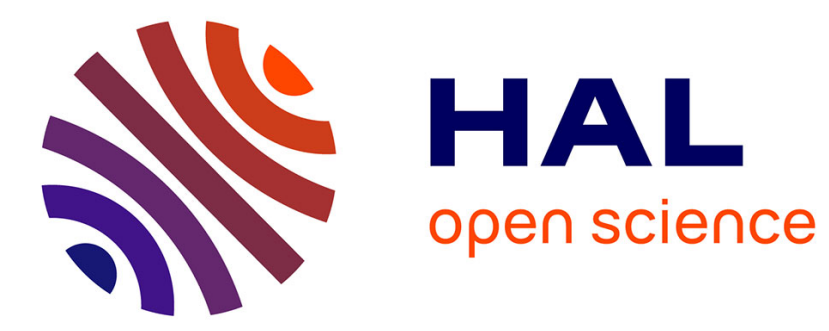

\title{
Granular flows down inclined channels with a strain-rate dependent friction coefficient. Part II : cohesive materials
}

\author{
Alain de Ryck
}

\section{- To cite this version:}

Alain de Ryck. Granular flows down inclined channels with a strain-rate dependent friction coefficient. Part II : cohesive materials. Granular Matter, 2008, 10 (5), p.361-367. 10.1007/s10035-008-0106-2 . hal-01846924

\author{
HAL Id: hal-01846924 \\ https://hal.science/hal-01846924
}

Submitted on 8 Nov 2019

HAL is a multi-disciplinary open access archive for the deposit and dissemination of scientific research documents, whether they are published or not. The documents may come from teaching and research institutions in France or abroad, or from public or private research centers.
L'archive ouverte pluridisciplinaire HAL, est destinée au dépôt et à la diffusion de documents scientifiques de niveau recherche, publiés ou non, émanant des établissements d'enseignement et de recherche français ou étrangers, des laboratoires publics ou privés. 


\title{
Granular flows down inclined channels with a strain-rate dependent friction coefficient. Part II: cohesive materials
}

\author{
Alain de Ryck
}

\begin{abstract}
A strain-rate dependent rheology for granular materials with a constant cohesion, is proposed and applied in the case of a flow down an inclined channel. The results obtained show that a plug flow zone appears below the free surface and show that low cohesion may affect drastically the flow behaviour when the inclination of the channel is close to the repose angle. These results give also a basic understanding of $h_{\text {stop }}$, the depth of the remaining granular layer on an inclined channel, treating dilatancy like a cohesion-like behaviour.
\end{abstract}

Keywords Granular materials - Cohesive materials · Surface flows

\section{Introduction}

Humid or fine granular materials exhibit a reduced flowability as soon as the attractive forces between particles become greater than their weight. The consideration of their flow behaviour is of significance for a better design of industrial processes or for the understanding of some geological flows like humid snow avalanches.

To study quantitatively the effect of cohesion in the particular case of a flow on an inclined channel, we present

\footnotetext{
A. de Ryck ( $\varangle)$

École des Mines d'Albi, UMR CNRS 2392, Route de Teillet, 81000 Albi, France

e-mail: deryck@enstimac.fr

A. de Ryck

Centre for Simulation and Modelling of Particulate Systems,

School of Materials Science and Engineering,

The University of New South Wales,

Sydney, NSW 2052, Australia
}

here an extension of the semi-analytical description given in the Part I joint paper [4]. The granular material exhibits a coefficient of friction which depends on the inertial number $I=\frac{|\dot{\gamma}| d}{\sqrt{P / \rho}}[2,7]$, and a residual constant cohesion $c$ during flow. $P=\sigma_{i i} / 3$ is the mean pressure, $\sigma$ is the stress tensor. $d$ is the diameter of the particles, $\rho$ the bulk density, $\dot{\gamma}$ is the strain rate tensor and $|\dot{\gamma}|=\sqrt{\dot{\gamma}_{i j} \dot{\gamma}_{i j} / 2}$ its second invariant.

This may be considered as a first simplified approach for cohesive materials since the cohesion term is not expected to be constant. The strength at yield is dependent on the consolidation of the material [12], and close to zero when the system is at the so-called critical state [13]. Recent numerical experiments by Rognon et al. [10] suggest an another rheology for cohesive materials, where there is no cohesion term, but the friction coefficient increases dependently on two numbers, the inertial number $I$, and the cohesion number $\eta=\frac{f_{\max }}{P d^{2}}$, where $f_{\text {max }}$ is the maximal value taken by the attractive forces between particles.

With this constant cohesion term, the stress-strain relation then writes:

$\sigma_{i j}=-P \delta_{i j}+(c+\mu(I) P) \frac{\dot{\gamma}_{i j}}{|\dot{\gamma}|}$.

We set-up the equations for a steady-state gravitational flow along an incline in Sect. 2, then the constant friction solution is discussed in Sect. 3, and the general solution in Sect. 4.

\section{Mathematical formulation}

If we consider the same geometry than in the non-cohesive case (see Fig. 1 in [4]), the Navier-Stokes equations for a steady-state flow writes: 


$$
\begin{aligned}
\vec{\nabla} & \cdot\left(\begin{array}{ccc}
P & 0 & (c+\mu P) \sin \alpha \\
0 & P & (c+\mu P) \cos \alpha \\
(c+\mu P) \sin \alpha & (c+\mu P) \cos \alpha & P
\end{array}\right) \\
& =-\rho g\left(\begin{array}{c}
0 \\
\cos \theta \\
\sin \theta
\end{array}\right),
\end{aligned}
$$

where $\alpha$ is the local angle made by the iso-velocity line with respect to the $x$-axis.

These equations lead to a hydrostatic mean pressure:

$P=\rho g \cos \theta y$,

and to a momentum equation along the $z$-axis which writes:

$\frac{\partial}{\partial x}((c+\mu P) \sin \alpha)+\frac{\partial}{\partial y}((c+\mu P) \cos \alpha)-\rho g \sin \theta=0$,

where $q=P / \rho g$.

In the following sections, this system will be integrated with a non-slip condition at the walls ( $w=0$ for $x= \pm a$ ) and a free surface $\left(\left.\alpha\right|_{y=0}=\frac{\pi}{2}\right)$. First, the case of a constant coefficient of friction will be considered (Sect. 3), then the more general case will be developed (Sect. 4).

\section{Constant friction}

With the hypothesis of constant friction, Eq. 4 reduces to:

$$
\left(\ell_{s}+y\right)\left(\cos \alpha \frac{\partial \alpha}{\partial x}-\sin \alpha \frac{\partial \alpha}{\partial y}\right)+\cos \alpha-R=0
$$

where

$$
\ell_{s}=\frac{c}{\mu \rho g \cos \theta}
$$

is a cohesive length and $R=\frac{\tan \theta}{\mu}$. This equation can be integrated as an ordinary differential equation along a $s$ parametred curve:

$$
\begin{aligned}
& \frac{d x}{d s}=\cos \alpha\left(\ell_{s}+y\right), \\
& \frac{d y}{d s}=-\sin \alpha\left(\ell_{s}+y\right), \\
& \frac{d \alpha}{d s}=R-\cos \alpha .
\end{aligned}
$$

Such a curve can be integrated from the point $(0, h)$ for which we have $\alpha=0$.The integration of the system (Eqs. 7, 8 and 9), using the same method than presented in the Annex 1 of the companion paper [4], leads to the following parametric equations for the iso-velocity curve:

$$
\begin{aligned}
& x=\left(h+\ell_{s}\right) \frac{s+\frac{R}{k} \sin (k s)}{1+R}, \\
& y+\ell_{s}=\left(h+\ell_{s}\right) \frac{R+\cos (k s)}{1+R},
\end{aligned}
$$

where $k=\sqrt{R^{2}-1}$.

The solution is similar to the one obtained for a non cohesive powder, with the exception that the $y$-co-ordinates are shifted by $\ell_{s}$. This leads to more flow patterns.

If the slope of the incline is less than $\mu$, then $k$ is a pure imaginary number and the iso-velocity is curved downwards, then collide the wall, and the conclusion is that $w=0$ everywhere.

If the slope of the incline is greater than $\mu$, then the isovelocity lines are curved upwards. They touch the free surface if $y=0$ is solution of the Eq. 11, which leads to the condition: $\frac{h}{\ell_{s}} \leq \frac{2}{R-1}$. In such the case, the iso-velocity lines arrive at the surface with an angle $\left.\alpha\right|_{y=0}$ given by:

$\left.\cos \alpha\right|_{y=0}=1-\frac{h}{\ell_{s}}(R-1)$.

From this equation, we conclude that only the iso-velocity line starting at the depth $h=\frac{\ell_{s}}{R-1}$ presents no stresses at the free surface. Then there is no steady-state flow solutions. We even not have the solution which consists to the sliding of two rigid bodies at the interface given by this particular iso-velocity line since the forces are not balanced.

\section{Strain-rate dependent friction coefficient}

With an $I$-dependent coefficient of friction, the parametred equations for an $x$-symmetric iso-velocity line passing through the point $(0, h)$ may be obtained the same way than for the non-cohesive case [4]. This leads to:

$$
\begin{aligned}
& \frac{d x}{d s}=\cos \alpha\left(\mu_{s} \ell_{s}+\mu y\right), \\
& \frac{d y}{d s}=-\sin \alpha\left(\mu_{s} \ell_{s}+\mu y\right), \\
& \frac{d \alpha}{d s}=\tan \theta-\mu \cos \alpha-\frac{d \mu}{d I} I\left(B \frac{y}{h}-\frac{1}{2} \cos \alpha\right) .
\end{aligned}
$$

where $B=h \frac{\phi^{\prime}(h)}{\phi(h)}, \phi$ being the velocity gradient at $x=0$ along the $y$-axis and the prime a derivation towards $y$.

These equations may be integrated once to obtain:

$$
\begin{aligned}
& \frac{d x}{d s}=\mu_{s} \ell_{s}+\mu(h) h+(y-h) \tan \theta-\frac{B}{h} \int_{h}^{y} \frac{d \mu}{d I} I y d y \\
& \left(\frac{d y}{d s}\right)^{2}=\left(\mu_{s} \ell_{s}+\mu y\right)^{2}-\left(\frac{d x}{d s}\right)^{2} .
\end{aligned}
$$

In order to have an iso-velocity line going to free surface, we must have:

$$
\left.\frac{d x}{d s}\right|_{y=0}=0
$$


which gives the following value for $B$ :

$B=h \frac{h \tan \theta-\mu(h) h-\mu_{s} \ell_{s}}{\int_{h}^{0} \frac{d \mu}{d I} I y d y}$.

With $\ell_{s}=0$, we retrieve the result obtained in [4].

To attain the surface, the curvature of the iso-velocity line must be oriented upwards, which gives a maximum value $B_{\max }$ for $B$ given by:

$B_{\max }=\frac{1}{2}+\frac{\tan \theta-\mu(h)}{\left.\left(\frac{d \mu}{d I} I\right)\right|_{y=h}}$,

Equation 19 may be integrated in order to obtain the admissible velocity profiles. For the next steps of resolution of these equations, we will follow the same scheme developed in [4], with the same $\mu(I)$ relation:

$\mu(I)=\mu_{s}+\frac{\mu_{2}}{\frac{I_{o}}{I}+1}$,

with $I_{o}=0.28, \mu_{s}=0.38$, and $\mu_{2}=0.64$. The equations are made dimensionless by scaling the lengths with $\lambda=$ $\left(\frac{\phi d}{I_{o} \sqrt{g \cos \theta}}\right)^{2}$, leading to $\frac{I_{o}}{I}=\sqrt{Y}$, where $Y=\frac{y}{\lambda} \cdot \psi$ is defined as in [4] by $H=e^{-2 \psi}$. We then have $B=\frac{1}{2}+\frac{d \psi}{d \ln h}$.

The Eq. 19 gives an ordinary differential equation for $\psi$, which has been integrated for several values of the parameter $\frac{h}{\ell_{s}}$. A set of results are given in Fig. 1 in continuous lines. The dashed line borders a zone where there is no valid solution since $B>B_{\max }$ in it. We observe that the behaviour for large $h$ tends to the one computed for a non-cohesive powder: $\psi$ tends to a constant $\psi^{*}$ and therefore the velocity gradient at the centerline behaves like a Bagnold one $(\phi \propto-\sqrt{h})$. This means that the behaviour of large scale flows of cohesive materials (compared to the cohesive length $\ell_{S}$ ) is similar to the flow of a non-cohesive material.

\subsubsection{Plug flow}

For small $h, \psi$ diverges positively or negatively. In the first case, it leads to non-physical solutions since the velocity gradient diverges for a finite value of the depth. In the second case, the velocity gradient $\phi \propto \sqrt{h} e^{\psi}$ tends to zero for a finite value of $h$. These solutions are the $\psi$-lines which are located between the thick and thin dashed lines in Fig. 1. Such a plug flow behaviour has been recently observed in numerical and physical experiments of cohesive granular flows down an incline [1,11].

These feasible solutions end to a plug zone at a depth $h_{\text {plug }}$, which is bounded by:

$$
1<\frac{h_{\text {plug }}}{\ell_{S}}(R-1)<\frac{2-\frac{m}{2}}{1-\frac{m}{2}} .
$$

The upper limit is obtained by looking the limit behaviour of the equality $B=B_{\max }$, when $\phi$ goes to zero. It is written

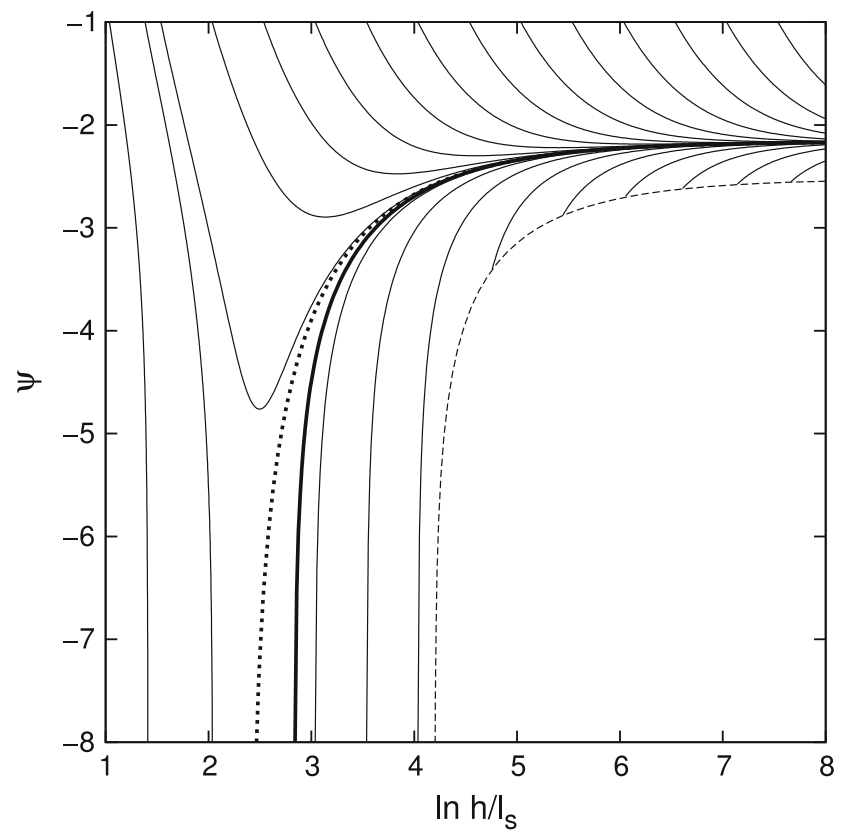

Fig. $1 \psi \propto \log \frac{\phi}{\sqrt{h}}$ versus $\log \left(\frac{h}{\ell_{s}}\right)$, for $\theta=22.6^{\circ}$ and $\mu$ given by Eq. 21. Continuous lines profiles for different initial conditions. When $h \rightarrow \infty$, then we recover the non-cohesive behaviour and $\psi \rightarrow \psi^{*}$. Thin dashed line borderline for the existence of iso-velocity lines curved upwards; the asymptote for this thin dashed line is $\frac{h}{\ell}=3 /(R-1)$. Thick dashed line limit between positive or negative divergence for $\psi$; the asymptote for this thick dashed line is $\frac{h}{\ell_{s}}=1 /(R-1)$. Thick line equilibrated profiles

in the generic case where $m$ is the power-law exponent characterizing the behaviour of the coefficient of friction versus the strain rate when this strain rate tends to zero $(\mu(I) \underset{I \rightarrow 0}{\sim}$ $\left.\mu_{s}+I^{m}\right)$. In the case of Eq. 21 [7], or from the observations by Da Cruz et al. [2] or Rognon et al. [10], we have $m=1$. Hatano et al., from numerical results and theoretical considerations, derive $\mu(I) \sim I^{\frac{1}{5}}[6]$.

Among these solutions, there is only one for which the force balance at the boundary of the plug zone is respected. The Appendix gives the value $h_{\text {plug }}$ of the plug flow depth for this unique solution. It scales with the cohesive length $\ell_{s}$ and only depends on the ratio $R$ between the slope of the incline and the static coefficient of friction. Figure 2 gives this depth $h_{\text {plug }}$, scaled by $\frac{\ell_{s}}{R-1}$ versus the relative difference between the slope and the static coefficient of friction (continuous line). In the same figure, the dashed line gives the ratio between the depth and the half-width $x_{\text {plug }}$ of this plug zone.

The asymptotic behaviour for the depth of the plug zone when the inclination angle tends to the repose angle is: $h_{\text {plug }} \underset{R \rightarrow 1}{\longrightarrow} \frac{3}{2} \frac{\ell_{s}}{R-1}$. There is also a maximum limit for the incline slope above which there is no more equilibrated solution within the range given by Eq. 22. Figure 3 gives the limit ratio $R_{\text {lim }}$ we obtain for each value of $m$ between 0 and 2 (continuous line), and the plug depth scaled by $\frac{\ell_{s}}{R-1}$ (dashed line). We observe in this figure a divergence for 


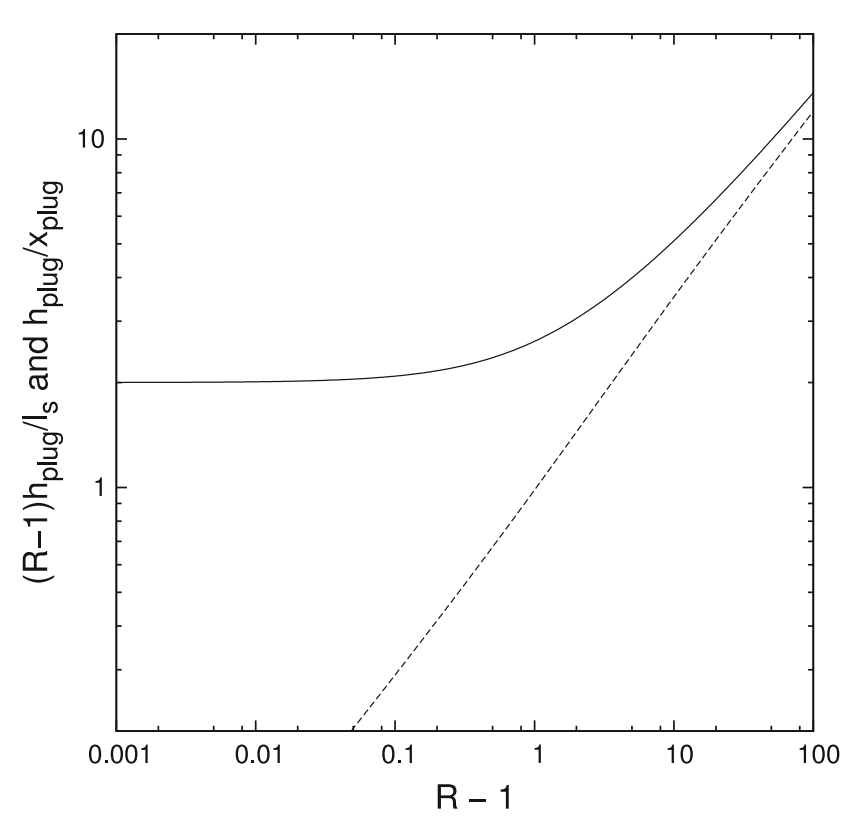

Fig. 2 Continuous line depth of the plug zone $h_{\text {plug }}$, scaled by $\frac{\ell_{s}}{R-1}$ versus the relative difference between the slope and the static coefficient of friction; Dashed line $h_{\text {plug }}$ scaled by the half width of the plug zone $x_{\text {plug }}$

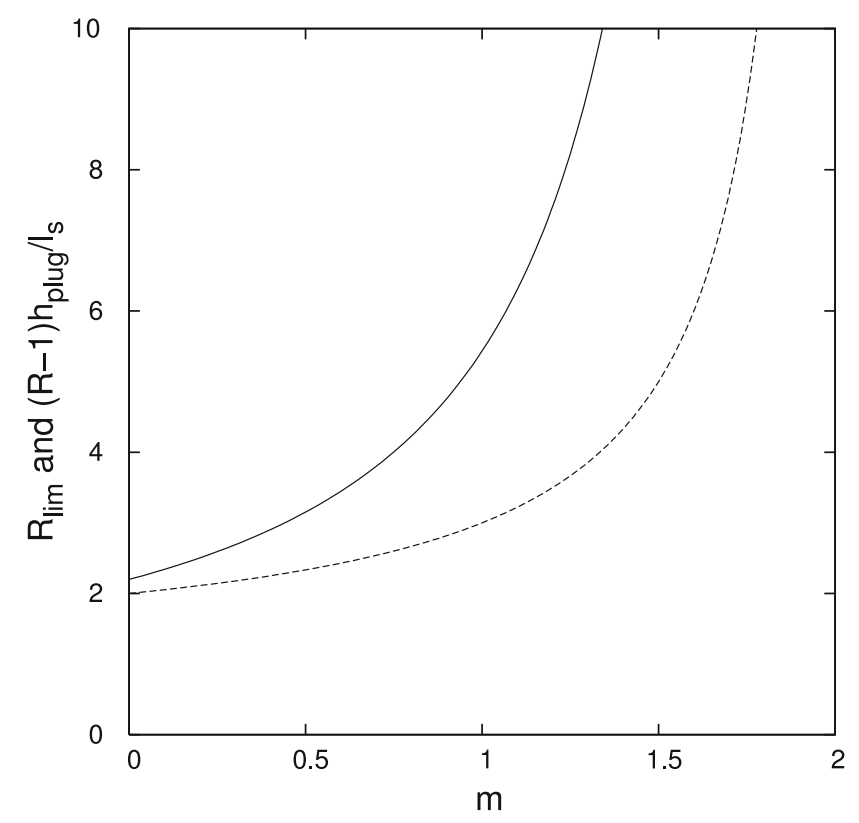

Fig. 3 Maximal value for steady-state flow $R_{\text {lim }}$ for the slope scaled by the static coefficient of friction versus the exponent $m$ (continuous

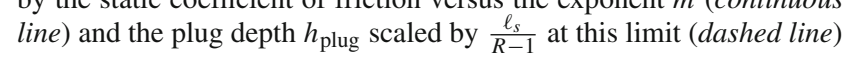

$m=2$, which corresponds to a shear friction going to a constant value when the mean pressure goes to zero $(\mu P \underset{P \rightarrow 0}{\longrightarrow} c s t)$. The coefficient of friction diverges at low pressure and the the friction shear stress presents a threshold value. The other constraint for the incline slope is that it should remain below $\mu_{2}$, the maximum value of $\mu(I)$.

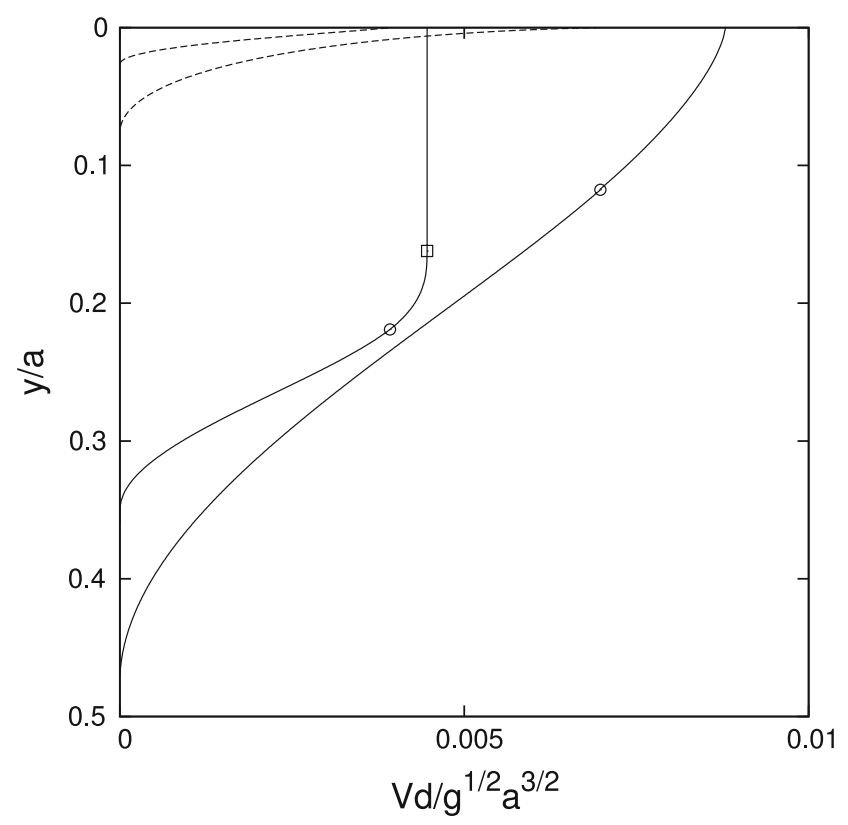

Fig. $4 \psi$ Vertical velocity profile at $x=0$ for $\theta=22.6^{\circ}, \mu_{s}=$ $\arctan \left(20.9^{\circ}\right)$ and $\mu_{2}=\arctan \left(32.6^{\circ}\right)$. Continuous lines non-cohesive case (lower) and cohesive case (upper, $\ell_{s}=0.02 a$ ). Dashed lines: Slip velocity at wall: non-cohesive case (lower) and cohesive case (upper). The circles gives the beginning of the matching $(w=w( \pm a, 0))$. The square gives the end of the plug flow

\subsubsection{Matching with slippage at the walls}

Finally, the flow layer composed by the iso-velocity lines going to the free surface is matched with the flow layer formed by the iso-velocity lines going to the walls, like in the non-cohesive case [4]. We have also chosen the rough walls boundary conditions to obtain the flow profile given in Fig. 4, which compares the depth velocity profiles at the center (continuous lines) and at the walls(dashed lines). The lower curves describe the non-cohesive behaviour and the upper curves are for a cohesion corresponding to a cohesive length $\ell_{s}=0.02 a$, where $a$ is the half-width of the channel. The inclination of channel is $\theta=22.6^{\circ}$ and $\mu(I)$ is given by Eq. 21.

The consequence of the cohesion is to reduce the velocities and to create a plug flow under the surface. This behaviour is noticeable including for cohesive lengths small compared to the size of the channel. In the case of Fig. 4, the cohesive length $c / \rho g$ is more than one hundred times lower than the width of the channel.

\section{Discussion}

The introduction of a constant cohesion in the rheology described in [7] has led to modified velocity profiles compared to the ones obtained with no cohesion. Below the free surface, 
we have a plug flow of depth proportional to the length $\frac{c}{\rho g}$, with a constant of proportionality $\left(\cos \theta\left(\tan \theta-\mu_{s}\right)\right)^{-1}$ which diverges when the slope of the incline approaches the angle of repose.

\subsection{Remaining cohesion during flow}

The constant cohesion hypothesis is a crude approximation since it is observed a huge decrease of the strength when the flow starts and the existence of a remaining cohesion during flow remains to be assessed [13]. To check that point, we have performed some yield and steady-state shear experiments of dry and wetted glass beads of diameter $200 \mu \mathrm{m}$. The beads have been wetted by poly-ethylene glycol (PEG) of molecular weight $200 \mathrm{~g}$ with a volume ratio of $1.5 \%$. In our experiments, the cohesion number $\eta$ is $<5$.

The shear stress at steady-state versus the normal stress is displayed on Fig. 5 for both beads. The dry beads (crosses) have a cohesion-less behaviour with a shear stress proportional to the normal stress. The wet beads, which have a cohesion around $500 \mathrm{~Pa}$ at yield (not shown) do not present a measurable cohesion at steady-state (see circles in Fig. 5). There is a slight shift between the two curves indicating that the friction coefficient of the wetted beads is higher than the friction coefficient of the dry ones. We also observe a slight curvature at low stresses on the $\tau-\sigma$ curve for the wetted beads indicating that, if there is a remaining cohesion, its order of magnitude is lower than $100 \mathrm{~Pa}$. Such a low value gives cohesive lengths $\ell_{s}$ around a few millimeters and we do not expect consequences on the flow behaviour when this flow occurs at a much larger scale. Nevertheless, if the depth of the plug flowscales with $\ell_{s}$, the coefficient of proportionality $1 /\left(\tan \theta-\mu_{s}\right)$ diverges when the inclination of channel approaches the angle of repose. The consequence is that very small cohesion may have a measurable effect on the flow at the threshold of sliding on an incline. In Fig. 4, the surface velocity is divided by a factor of 2 for a cohesive length 70 times lower than the width of the channel and $1.7^{\circ}$ of difference between the repose angle and the channel inclination. This situation corresponds to a $20 \mathrm{~cm}$ width channel for our wetted glass beads. As a conclusion, flows in inclined channels are very sensitive to low cohesions when their slopes are close to the static coefficient of friction, the so-called repose angle. This behaviour may become a convenient tool to estimate them.

\subsection{Cohesion-like dilatancy}

This constant cohesion modeling may also be applied for a non-cohesive material with dilatancy effects in order to obtain $h_{\text {stop }}$, the thickness of the remaining granular layer on an incline [9]. This cohesion-like term should be the specific energy dissipated to maintain the dilated state and scales:

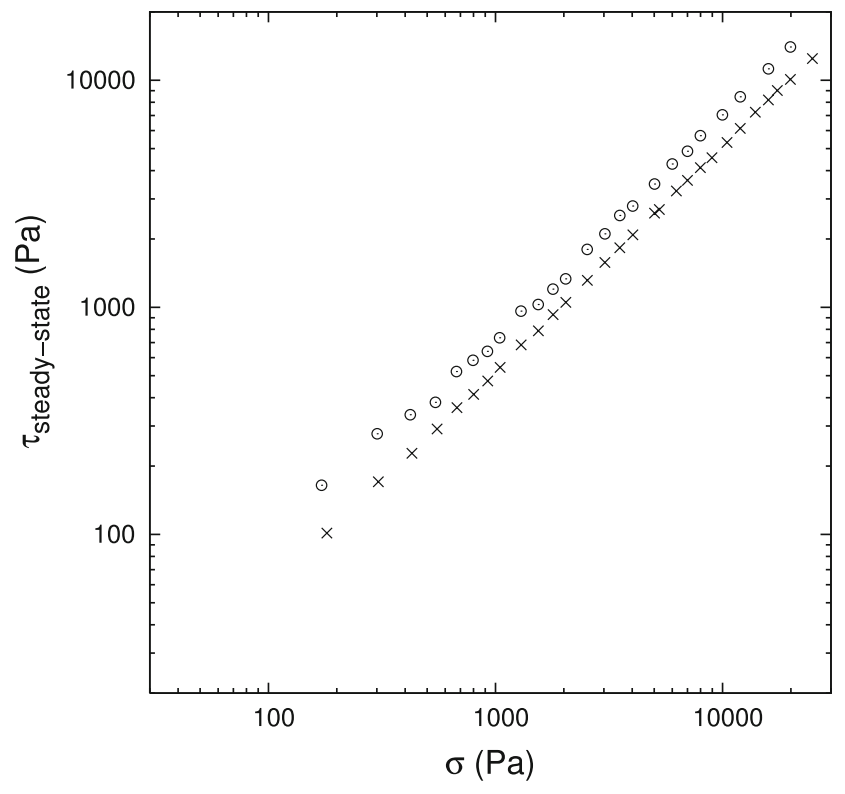

Fig. 5 Steady-state shear stress versus the normal stress for dry (crosses) and wet glass beads (circles) of diameter $200 \mu \mathrm{m} .15 \mathrm{ml} / \mathrm{l}$ of poly-ethylene glycol of molecular weight $200 \mathrm{~g}$ have been used for these experiments

$c \simeq \rho g \cos \theta \Delta$,

where $\Delta$ is the displacement of the barycentre normal to the flow direction. A rough estimate of this length is that it scales like the size of the particles and should vanish when the slope of the incline approaches the maximal value for $\mu(I)$.

To validate this hypothesis, we have calculated the quantity:

$\frac{\Delta}{d}=\mu_{s} \frac{h_{\text {stop }} / d}{h_{\text {plug }} / \ell_{s}}$

In Eq. 24, the numerator is taken directly from the experimental data by Forterre and Pouliquen [5] and Daerr and Douady [3]. The denominator comes from Eq. 26 in the Appendix, with $R$ being built from the experimental slope of the incline divided by $\mu_{s}$, the coefficient of friction when $h_{\text {stop }}$ goes to infinity.

The results are plotted in Fig. 6 versus the difference between the inclination angle and its maximal value. The error bars come from the fact that the determination of $\mu_{s}$ is within $1^{\circ}$ of angle precision. We observe first that $\Delta$ is experimentally of order the size of the beads used. We also observe that the behaviour, for inclination angles close to the maximal value, is similar for both experiments. Despite the approximations used, we obtain a good evaluation for the minimal thickness of the remaining layer of particles in an incline with this cohesion-like behaviour for dilatant granular 


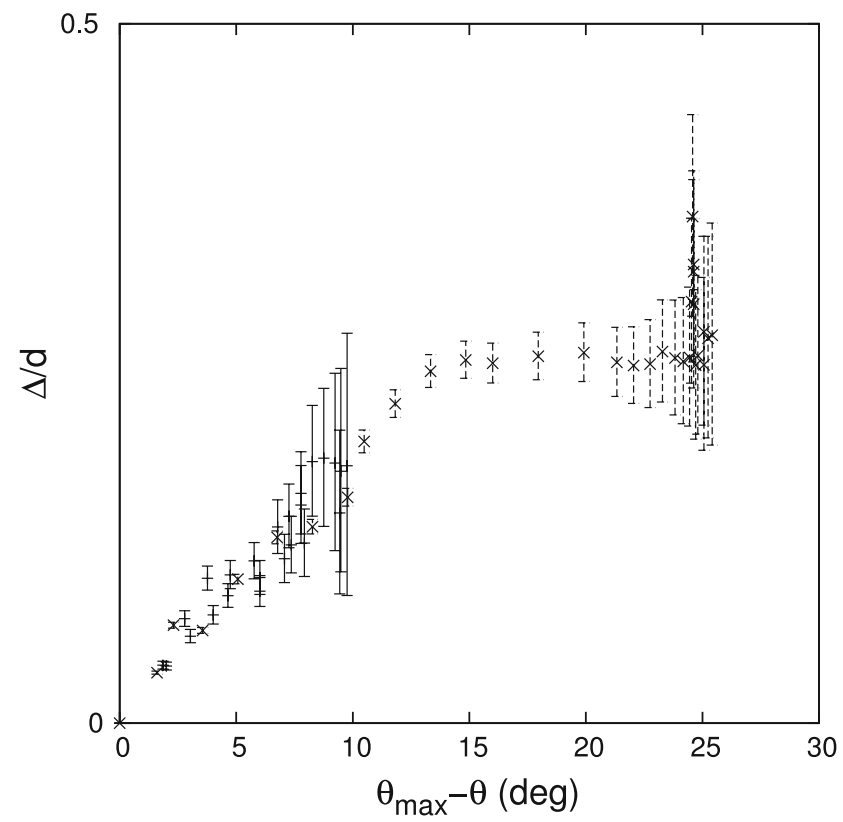

Fig. $6 h_{\text {stop }} / d$ experimental data by Forterre and Pouliquen [5] (plus symbol) and Daerr and Douady [3] (cross) scaled by $h_{\text {plug }} / \ell_{s}$ versus the difference between the inclination angle and its maximal value. The error bars represents an incertitude of $1^{\circ}$ in the determination of $\mu_{s}$

materials. The fact that the rheological model is written for non-dilatant granular materials has a low impact because the flow is non-confined, then the mean pressure remains similar with or without dilatancy and the previous heuristic arguments remain valid.

A final remark is that a flow of non-cohesive material should also exhibit a plug flow below the free surface, but such a behaviour has not been reported. To explain that point, we may calculate the plug flow depth at the onset of flow in the case of a coarse wall.

At the onset of flow, the plug invades all the width of the channel, therefore $x_{\text {plug }}=a$, and the Eqs. 27 and 26 give implicitely the depth of the plug zone $h_{\text {onset }}$, scaled by the size $d$ of the particles, using $\ell_{s}=0.25 \frac{d}{\mu_{s}}$. The result is shown in Fig. 7 using Eq. 21 and $\frac{a}{d}=5,50,500$ and 5,000 from bottom to top. We observe that, as soon as the inclination angle exceed by $3^{\circ}$ the onset inclination angle, the depth of the plug flow is lower than a tens particles. As a conclusion, this plug flow, for a non-cohesive granular material remains small, except close to the onset of flow. It may be added that the transition between the plug and the flow below it may be softened by some creep motion, as observed by Komatsu et al. [8]. Such a creep not taken into account by the rheology employed.

\section{Concluding remarks}

The introduction of a constant cohesion in the rheology described by Eq. 1 leads to a modified flow pattern compared to

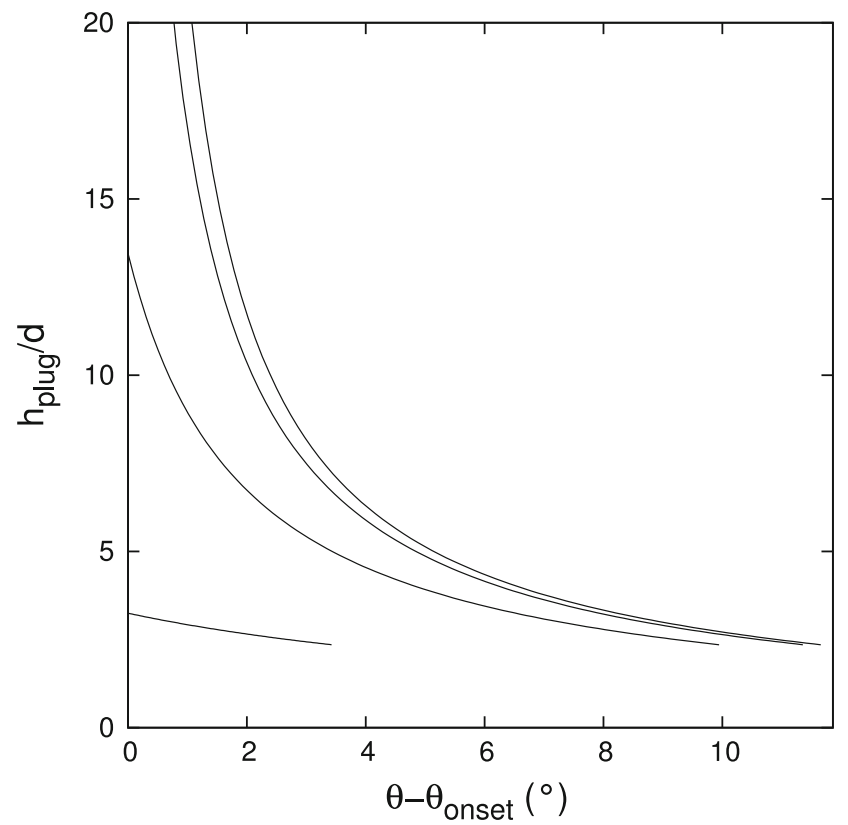

Fig. $7 h_{\text {plug }} / d$ versus the difference between the inclination angle and its value at the onset of flow. $\mu$ is given by Eq. 21 . From bottom to top, the width of the channel is $10,100,1,000$ and $10,000 \mathrm{~d}$

the non-cohesive case. We obtain a plug flow under the free surface. Its size scales like the cohesive length $\ell_{s}$, given by Eq. 6, times the inverse of the relative ratio between the slope of the incline and the static coefficient of friction. In order to obtain a flow, the depth and width of the powder bed must be greater than this typical length.

The flow behaviour is then highly sensitive to small cohesion values when the inclination angle is close to the angle of repose.

From the observation of this behaviour, we hypothesize that the dilatancy effects of non-cohesive materials may be taken into account by the rheological model with a cohesion whose order of magnitude corresponds to a cohesive length of the same order of size than the particles. This prohibits flows at a size scale lower than the size of the particles and gives $h_{\text {stop }}$, the thickness of the remaining layer of grains on an incline, to diverge when the inclination angle approaches the angle of repose.

Acknowledgments The author takes the pleasure to thank Haiping Zhu and Aibing Yu for fruitful discussions.

\section{Appendix: Determination of the plug zone depth}

When the velocity gradient $\phi$ tends to zero, the iso-velocity lines going to the free surface tends to the solution for a constant coefficient of friction (Eqs. 10 and 11) (continuous line in Fig. 8), when the angle $\alpha$ remains lower than $\frac{\pi}{2}$. 


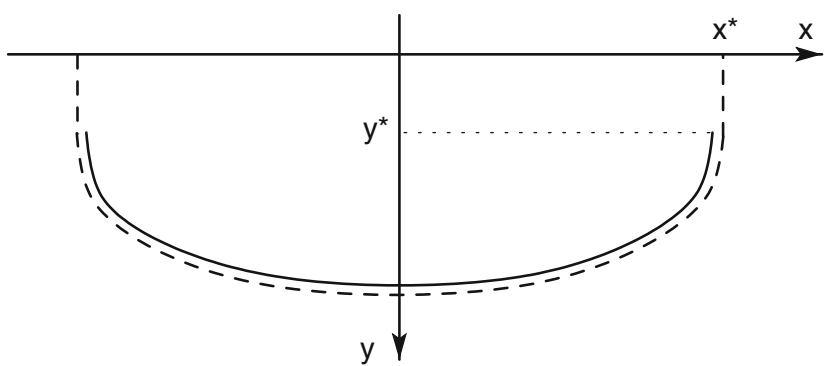

Fig. 8 Sketch of the constant coefficient of friction solution (continuous line) and $I$-dependent coefficient of friction limit solution when the velocity gradient $\phi$ tends to zero (dashed line)

The constant friction solution attains $\alpha=\frac{\pi}{2}$ at a point $\left(x^{*}, y^{*}\right)$ below the free surface for $h>\frac{\ell_{s}}{R-1}$. This point is attained for $s=s^{*}$, with $\cos \left(k s^{*}\right)=-1 / R$.

After that, the iso-velocity lines tends to reach the free surface like a straight line normal to it, as sketched in Fig. 8 in dashed line.

$h_{\text {plug }}$ is obtained by equating the shear force acting on the limit iso-velocity line with the weight in the $z$-direction. It writes:

$$
\begin{gathered}
\int_{0}^{s^{*}}\left(\ell_{s}+\left.y\right|_{c s t}\right)^{2} d s+\int_{0}^{y^{*}}\left(\ell_{s}+y\right) d y \\
-\left.\left.R \int_{0}^{s^{*}} y\right|_{c s t} \frac{d x}{d s}\right|_{c s t} d s=0,
\end{gathered}
$$

where the $c s t$-suffix means that we take the constant friction solution. This leads to the solution:

$h_{\text {plug }}=\frac{\ell_{s}}{R-1}\left(1+\sqrt{\frac{1+R^{2} s^{*}}{1+s^{*}}}\right)$.
The half-width of the plug is:

$x_{\text {plug }}=\ell_{s}\left(R+\sqrt{\frac{1+R^{2} s^{*}}{1+s^{*}}}\right) \frac{1+s^{*}}{R^{2}-1}$.

\section{References}

1. Brewster, R., Crest., G.S., Landry, J.W., Levine, A.J.: Plug flow and the breakdown of bagnold scaling in cohesive granular flows. Phys. Rev. E 72, 061301 (2005)

2. Da Cruz, F., Emam, S., Prochnow, M., Roux, J.N., Chevoir, F.: Rheophysics of dense granular materials: discrete simulations of plane shear. Phys. Rev. E 72, 021309 (2005)

3. Daerr, A., Douady, S.: Two types of avalanche behaviour in granular media. Nature 399, 241-243 (1999)

4. de Ryck, A., Ansart., R., Dodds, J.A.: Granular flows down inclined channels with a strain-rate dependent friction coefficient. Part I: non-cohesive materials. Granular Matter (2007). doi:10.1007/s10035-008-0105-3

5. Forterre, Y., Pouliquen, O.: Long-surface-wave instability in dense granular flows. J. Fluid Mech. 486, 21-50 (2003)

6. Hatano, T., Otsuki, M., Sasa, S.I.: Criticality and scaling relations in a sheared granular material. J. Phys. Soc. Jpn. 76(2), 023001 (2007)

7. Jop, P., Forterre, Y., Pouliquen, O.: A constitutive law for dense granular flows. Nature 441, 727-730 (2006)

8. Komatsu, T., Inagaki, S., Nakagawa, N., Nasuno, S.: Creep motion in a granular pile exhibiting steady surface flow. Phys. Rev. Lett. 86(9), 1757-1760 (2001)

9. Pouliquen, O., Renaut, N.: Onset of granular flows on an inclined rough surface: dilatancy effects. J. Phys. II Fr. 6, 923-935 (1996)

10. Rognon, P.G., Roux, J.-N., Wolf, D., Naaïm, M., Chevoir, F.: Rheophysics of cohesive granular materials. Europhys. Lett. 74(4), 644-650 (2006)

11. Rognon, P.: Rheology of cohesive materials-application to dense snow avalanches. Ph.D. Thesis (in french), École Nationale des Ponts et Chaussées (2006)

12. Rumpf, H.: Zur Theorie der Zugfestigkeit von Agglomeraten bei Kraftübertragung an Kontaktpunkten. Chemie Ingenieur Technik 42(8), 538-540 (1970)

13. Wood, D.M.: Soil behaviour and critical state soil mechanics. Cambridge University Press, Cambridge (1990) 